\title{
EVALUATION OF CHONDROPROTECTIVE EFFECTS OF HYALURONIC ACID IN A RAT MODEL OF OSTEOARTHRITIS
}

\author{
Noaman Ishaq, Shabana Ali*, Muhammad Waqar Aslam Khan*, Kulsoom Farhat*, Nausheen Ata*, Quratulain Mehdi* \\ Bakhtawar Amin Medical and Dental College, Multan Pakistan, *Army Medical College/National University of Medical Sciences (NUMS) Rawalpindi Pakistan
}

\begin{abstract}
Objective: To evaluate the chondroprotective effects of hyaluronic acid in a rat model of osteoarthritis.

Study Design: Laboratory based experimental study.

Place and Duration of Study: Department of Pharmacology, Army Medical College, Rawalpindi, in alliance with National Institute of health, Islamabad and Department of Pathology, Army Medical College Rawalpindi, from Apr to Jun 2019.

Methodology: Sixteen (16) rats of Sprague Dawley breed were procured in this study. Osteoarthritis was induced in right knee joint of rats by surgical resection of medial meniscus and anterior cruciate ligament. They were allocated into two (02) groups with eight (8) rats in both groups. Group-I was control group that was treated with $0.2 \mathrm{ml}$ intra articular saline once weekly for four weeks. While group-II was treatment group that was intra particularly administered with $0.2 \mathrm{ml}$ hyaluronic acid once weekly for four weeks. One week after the last dosage, gait pattern of the animals was scored. Then animals were sacrificed and a part of proximal tibia was obtained for histopathologic analysis.

Results: Mean gait score of control group and treatment group was $3.25 \pm 0.707$ and $1.00 \pm 0.756$ respectively with a statistically significant $p$-value of $<0.001$, while mean histopathological Modified Mankin score of control and treatment group was $11.5 \pm 1.195$ and $5.50 \pm 1.195$ respectively with a significant $p$-value of $<0.001$.

Conclusion: Intra articular viscosupplementation of hyaluronic acid in rat model of osteoarthritis resulted in improved gait pattern and histopathology.
\end{abstract}

Keywords: Chondroprotective effects, Hyaluronic acid, Osteoarthritis.

How to Cite This Article: Ishaq N, Ali S, Khan MWA, Farhat K, Ata N, Mehdi Q. Evaluation of Chondroprotective Effects of Hyaluronic Acid in A Rat Model of Osteoarthritis. Pak Armed Forces Med J 2021; 71(6): 2189-2193. Doi: https://doi.org/10.51253/pafmj.v71i6.3984

\footnotetext{
This is an Open Access article distributed under the terms of the Creative Commons Attribution License (https://creativecommons.org/licenses/by-nc/4.0/), which permits unrestricted use, distribution, and reproduction in any medium, provided the original work is properly cited.
}

\section{INTRODUCTION}

Osteoarthritis (OA), also termed as osteoarthrosis, is a degenerative joint disease with inflammatory component. It is a heterogeneous disease of the whole joint with progressive loss of hyaline cartilage, rapid growth of bone architecture and remodeling of joint structure. It is characterized by gradual deterioration of articular cartilage with concomitant structural and functional changes in the entire joint including capsule, synovium, ligaments, bursas, subchondral bone and periarticular muscles. ${ }^{1}$ Age related degenerative changes are the main cause of development of OA in old age while physical or mechanical trauma to joint is main contributor to development of OA in young people. ${ }^{2}$ It is clear from multiple studies and surveys that $\mathrm{OA}$ is the most common disease of joints as compared to Rheumatoid arthritis (RA) and other seronegative forms of joint diseases. ${ }^{3}$ OA puts down a huge burden on healthcare department and consumes a substantial sum of healthcare resources. It involves patient's multiple

Correspondence: Dr Noaman Ishaq, Department of Pharmacology, Bakhtawar Amin Medical and Dental College, Multan, Pakistan Received: 04 Mar 2020; revision received: 29 Apr 2020; accepted: 12 May 2020 visits to outpatient departments (OPDs), cost of medicine, hospitalization and sometimes surgical replacement of the affected joint. ${ }^{4}$ Various drug groups are used to lessen the severity of symptoms and to delay the progression of disease. Nonsteroidal anti-inflammatory drugs (NSAIDs), corticosteroids and viscosupplement substances are widely used drug groups in the treatment of OA. Hyaluronic acid (HA), a viscosupplement substance, is a major constituent of synovial fluid which helps to facilitate lubrication and shock absorption in joints. ${ }^{5}$ It is one of the many investigational molecules in the treatment of OA. Viscosupplementation with intra articular (IA) HA has positive outcome in pain alleviation and joint function improvement in OA. 6 Experimental and in vitro studies depicted chondroprotective efficacy of HA along with its complementary clinical results. Various physiological effects apparently lead to the mechanism by which HA employs its chondroprotective effects. It increases proteoglycan synthesis, lessens the generation and action of proinflammatory mediators and matrix metalloproteinases (MMP) and remodels the response of immune cells. HA has a distinct effect on inflammatory mediators including cytokines, proteases and prostagl- 
andins. HA also exhibits antioxidant effects in different systems anticipating protection of articular chondrocytes against the damage influenced by oxygen-derived free radicals. ${ }^{7}$

This rationale of this animal study was that as the HA is an investigational drug; we planned this project to detect its chondroprotective effects in a rat model of OA.

\section{METHODOLOGY}

This was a laboratory based experimental study that was carried out in department of Pharmacology and Therapeutics, Army Medical College, Rawalpindi in alliance with National Institute of Health (NIH), Islamabad and Department of Pathology, AMC. Ethics approval of this study was undertaken from ethical review committee of "Centre for Research in Experimental and Applied Medicine", (Referece 1tr dated May 3, 2019). Duration of animal intervention was two months from April to June 2019. Animals were kept in animal house of NIH Islamabad during the whole duration of the study.

Inclusion Criteria: Adult rats of Sprague Dawley breed, age 8-10 weeks and weight about 300-400 gram were selected.

Exclusion Criteria: Apparently sick animals were excluded.

Preliminary sixteen (16) adult rats were selected. Animals were retained in metal cage; temperature was kept $25 \pm 5^{\circ} \mathrm{C}$ with $40 \pm 5 \%$ humidity and 12 hours day $/$ night cycle. Free excess to clean drinking water and standard rodent diet adlibitum was ensured during the complete tenure of the study. OA was induced in right knee joint of all rats through surgery. Initially they were anesthetized with intraperitoneal injection of $5 \%$ Xylazine and 1\% Ketamine. ${ }^{8}$ After that, skin of the joint was shaved and disinfected with pyodine. It was followed by a para patellar incision on the medial side of the joint. Skin of the joint was retracted for complete exposure of the joint. Anterior cruciate ligament and medial meniscus were identified and transected. Skin of the joint was closed with surgical stapler after the completion of the procedure. Then animals were allowed to move freely in the cage for two weeks thereafter. ${ }^{9}$ They were assigned in two (2) groups, control group and treatment group, with eight (8) rats in each group thereafter. Intra articular (IA) drugs were administered in the right knee joint of the rats two weeks after the surgery. Rats of control group were injected with $0.2 \mathrm{ml}$ of Normal saline once weekly for 4 weeks.
While 0.2ml HA was administered in right knee joint of treatment group once weekly for four weeks. We waited for one week after the last dosage before scrutinizing the parameters. Gait pattern was analyzed according to an established scale of gait score. ${ }^{10}$ After gait scoring, animals were sacrificed by inhaled chloroform in a desiccator. ${ }^{11}$ Part of proximal tibia was cut by $5.5^{\prime \prime}$ angled bone cutter thereafter. Slides of proximal tissue of tibia were prepared with Eosin and Hematoxylin stains. These slides were scored using Modified Mankin Slide score. ${ }^{12}$

Data was analyzed using Statistical package for the social sciences (SPSS) version 25. Gait score and Modified Mankin score both parameters of this study were quantitative and thus compared through student t-test. The differences between two observations were considered statistically significant if the $p$-value was equal or less than $0.05(p \leq 0.05)$.

\section{RESULTS}

After one week of last dosage, hind paws of rats were stained with dense black ink and they were tempted by food to walk full length of A2 $(42 \times 60 \mathrm{~cm})$ size paper. Gait pattern of the rats was noticed with naked eye and footprint made by the right drug treated leg was compared with the left non-treated one to analyze weight bearing throughout the movement on the paper. Gait score of three, four and one rats of control group was 04,03 and 01 respectively while gait pattern of two rats of treatment group scored 0 , four rats scored 04 and two rats scored 02. Figure- 1 showed gait pattern of a rat of control group with 04 score while Figure-2 exhibited gait pattern of a rat of treatment group with 01 score.

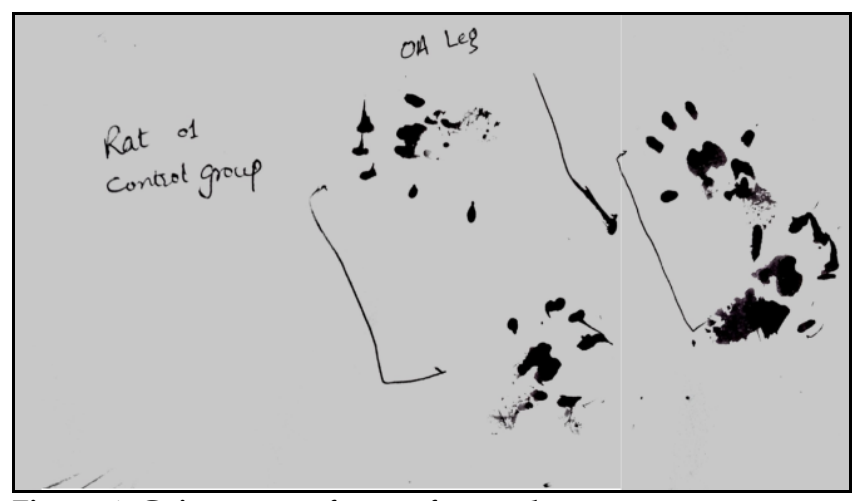

Figure-1: Gait pattern of a rat of control group.

Slides of knee joint of the rats were observed and analyzed under X100 and X400 lens. Histopathological changes of OA were scored according to Modified 
Mankin scoring system. Score of slides of control group was ranging from 10-13. Figure-3 depicted histopathological changes of a rat of this group with 13 score. Six out of eight slides showed marked while rest of two showed mild irregularity in perichondrium. One slide had marked, six had moderate and one had mild fibrosis of perichondrium. Six slides had moderate to marked while two slides had mild to moderate irregularity of cellular organization. All slides of this group showed moderate to marked increase in cellularity of chondrocytes. Five and three slides showed moderate and mild increase in chondrocyte clusters respectively. $10-20 \%$ chondrocyte necrosis was the feature of five slides while three slides exhibited $20-80 \%$ chondrocyte necrosis. Meanwhile all slides exhibited fibrinoid degeneration. Mean score of this group was $11.50 \pm 1.19$.
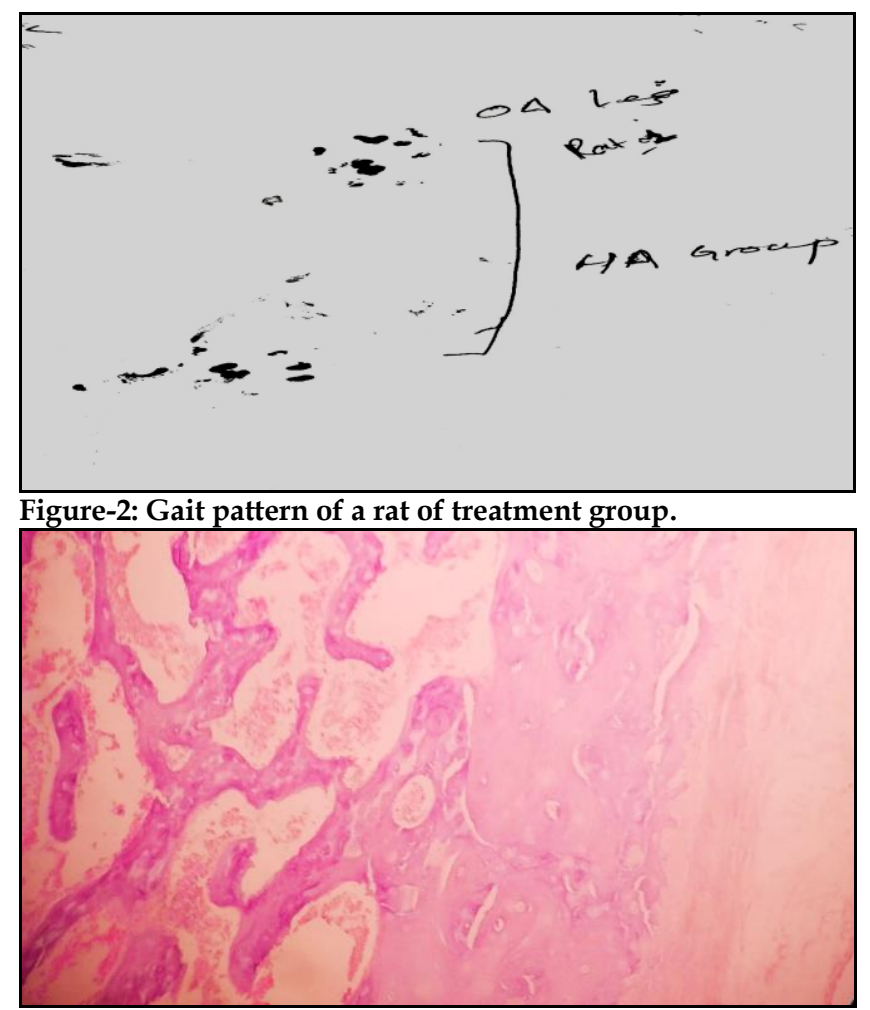

Figure-3: Photomicrograph of proximal tibia of a rat of Control group.

Slides of treatment group scored minimum ranging from 04-07. Mild to moderate irregularity of perichondrium was the feature of all the eight slides. Half of the slides showed no while half showed mild fibrosis of perichondrium. One slide had moderate to marked whereas seven slides had mild to moderate irregularity of perichondrium. Two slides had no while remaining five slides showed mild increase in cellularity of chondrocytes. Seven had mild while one had moderate increase in chondrocyte clusters. 10-20\% chondrocyte necrosis was the feature of all the slides. None of the slide in this group exhibited fibrinoid degeneration. Figure-4 was a photomicrograph of a slide of a rat of HA group with 05 score. It showed mild fibrosis of perichondrium with little increase in cellularity and $10-20 \%$ necrosis of chondrocytes.

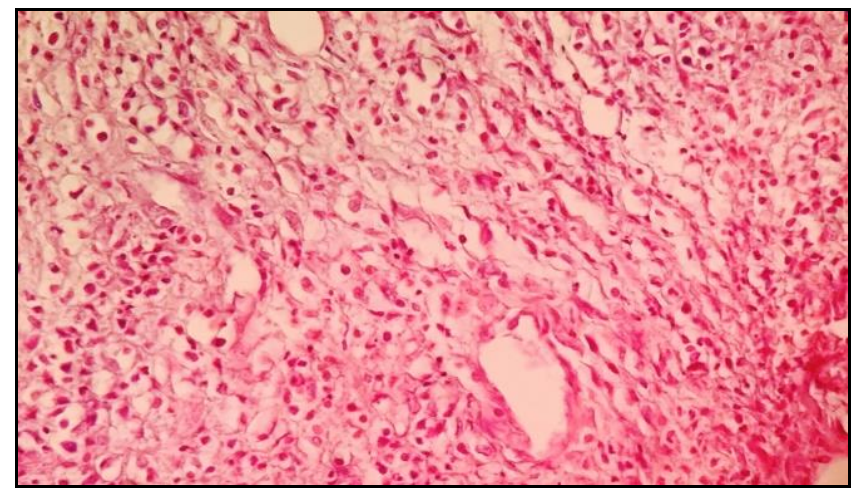

Figure-4: Photomicrograph of proximal tibia of a rat of treatment group.

The mean Gait score of control group and treatment group were $3.25 \pm 0.70$ and $2.25 \pm 0.75$ respectively with a significant $p$-value of $<0.001$ and Mean score of this group was $5.50 \pm 1.19$. When the histological score of both groups were compared, we found a highly significant $p$-value of $<0.001$, both time that confirmed the chondroprotective effects of HA in a rat model of OA as shown in the Table.

Table: Comparison of gait score and modified mankin score of histopathology.

\begin{tabular}{l|c|c|c}
\hline Parameters & $\begin{array}{c}\text { Control Group } \\
(\mathbf{n}=\mathbf{0 8})\end{array}$ & $\begin{array}{c}\text { Treatment } \\
\text { Group }(\mathbf{n}=\mathbf{0 8})\end{array}$ & $\begin{array}{c}\boldsymbol{p} \text { - } \\
\text { value }\end{array}$ \\
\hline $\begin{array}{l}\text { Mean of Gait } \\
\text { score }\end{array}$ & $3.25 \pm 0.70$ & $1.00 \pm 0.70$ & $<0.001$ \\
\hline $\begin{array}{l}\text { Mean of Modified } \\
\text { Mankin Score }\end{array}$ & $11.50 \pm 1.19$ & $5.50 \pm 1.19$ & $<0.001$ \\
\hline
\end{tabular}

\section{DISCUSSION}

Osteoarthritis (OA) is the most common manifestation of arthritis which becomes more severe as the people age. It is characterized by degeneration of cartilage, underlying bone and joint margin. ${ }^{13}$ It commonly involves the joints of knee, hip and hand. The universal burden of this ailment categorizes knee and hip OA as one of the common chronic disease. As life expectancy and ratio of aged population increases, prevalence of OA is also being increased. This disease has a higher incidence in old females as compared to males. Risk factors of OA are still evolving with some common factors include age, gender, weight, increased bio- 


\section{Chondroprotective}

mechanical loading of joints and genetics. ${ }^{14}$ Definite cure of OA is not available. Non-pharmacological interventions i.e. assistive devices (foot wear and knee braces), encouraging individuals to adapt healthy life style, dietary habits to control body weight and to commence moderate intensity exercises are the first preferences to manage a patient of OA. Pharmacological interventions are recommended when non-pharmacological interventions become ineffective. Different drug groups are used to alleviate the severity of symptoms and to delay the progression of disease. Nonsteroidal anti-inflammatory drugs (NSAIDs), corticosteroids and viscosupplements are widely used drug groups in the treatment of OA. Surgical interventions include arthroscopy, osteotomy and joint replacement can be an option for some individuals after the failure of pharmacological treatment. ${ }^{15}$ Animal models of chronic disease i.e. OA are created to find the efficacy of novel drugs. ${ }^{16}$ HA a novel drug, is a viscosupplement substance that is a natural component of synovial joint fluid. Chondroprotective effects of this drug are not yet established. This animal study was planned to find the chondroprotective effects of intra articular (IA) HA in a rat model of OA. Dosage of HA was selected from some recent research projects. After the completion of intervention protocol, parameters of both groups were analyzed and compared. There were statistically significant differences between gait score and modified mankin score of treatment group and control group that confirmed the chondroprotective effects of HA. Our results were in accordance to 2018 research of Zhenqinq et al. They Compared HA treated rabbit model of OA with disease control group and found a significant $p$-value of $<0.05 .{ }^{17}$ Similarly, in 2017 Akman et al created chondrocyte defect in knees of rats by $27 \mathrm{~mm}$ drill bit. They also concluded that HA exhibits chondroprotective efficacy when compared with the rats of control group ( $p$-value<0.001)..$^{18}$ In 2016 Tamura et al created antigen treated arthritis model of rats. They found a $p$-value of $<0.01$ when HA treated group was compared with vehicle treated group. ${ }^{19}$ Their findings also favor our results. Meanwhile research work of Ozkan et al in 2015 also declared the chondroprotective effects of HA as compared to saline treated group in rat model of OA with a $p$-value of 0.004 , results that are similar to our study. ${ }^{20}$

\section{ACKNOWLEDGEMENT}

Authors are thankful to Dr. Hussain Ali, Head of research animal Facility, National Institute of Health (NIH), Islamabad for his supervision regarding induction of oste- oarthritis and intra articular drug administration in rats. We also owe special thanks to Army Medical College, Rawalpindi, for providing us opportunity to carry out this research work.

\section{LIMITATION OF STUDY}

There were some limitations in our research project, as we did not assess local as well as systemic adverse effects of IA administration of HA. Similarly, there was a gap of 02 weeks between surgical induction of $\mathrm{OA}$ and first dosage administration. Delay of four to eight weeks may lead to induction of severe form of OA.

\section{CONCLUSION}

As compared to saline treated group, intra articular HA exhibits chondroprotective effects in a rat model of OA. It is suggested that further in vitro, animal and human studies should be carried out to strengthen our results.

\section{Conflict of Interest: None.}

Disclosure: A special gratitude of thanks to National University of Medical Sciences, Rawalpindi for funding our project.

\section{Authors' Contribution}

NI: Study design, animal intervention, SA: Study design \& biostatistics, MWAK: Biostatistics, KF: Results complitation, NA: Data analysis, QM: Study design \& biostatistics.

\section{REFERENCES}

1. Fu K, Robbins SR, McDougall JJ. Osteoarthritis: the genesis of pain. Rheumatol 2018; 57(4): 43-50.

2. Loeser RF, Collins JA, Diekman BO. Ageing and the pathogenesis of osteoarthritis. Nature Rev Rheumatol 2016; 12(7): 412-420.

3. Mobasheri A, Batt M. An update on the pathophysiology of osteoarthritis. Ann Phys Rehabil Med 2016; 59(5-6): 333-339.

4. 4.Vina ER, Kwoh CK. Epidemiology of osteoarthritis: literature update. Cur Opin Rheumatol 2018; 30(2): 160-167.

5. Leighton R, Fitzpatrick J, Smith H, Crandall D, Flannery CR Conrozier T. Systematic clinical evidence review of NASHA (Durolane hyaluronic acid) for the treatment of knee osteoarthritis. Open Access Rheumatol 2018; 10(1): 43-54.

6. Altman R, Bedi A, Manjoo A, Niazi F, Shaw P, Mease P. Antiinflammatory effects of intra-articular hyaluronic acid: a systematic review. Cartil 2019; 10(1): 43-52.

7. Kim SH, Park KW, Kim JM, Ho MJ, Kim HT, Song SH, et al. Pharmacokinetics and four-week repeated-dose toxicity of hyaluronic acid and ketorolac combination following intra-articular administration in normal rats. Regul Toxicol Pharmacol 2019; 102(2): 79-89.

8. Saadat E, Shakor N, Gholami M, Dorkoosh FA. Hyaluronic acid based micelle for articular delivery of triamcinolone, preparation, in vitro and in vivo evaluation. Int J Pharmac 2015; 489(1-2): 218225.

9. Sudirman S, Ong AD, Chang HW, Kong ZL. Effect of fucoidan on anterior cruciate ligament transection and medial meniscectomy induced osteoarthritis in high-fat diet-induced obese rats. Nutrients 2018; 10(6): 686-699.

10. Kumar A, Bendele AM, Blanks RC. Sustained efficacy of a single intra-articular dose of FX006 in a rat model of repeated localized knee arthritis. Osteoarthritis Cartil 2015; 23(1): 151-160. 


\section{Chondroprotective}

11. Asefi S, Seifi M, Fard GH, Lotfi A. Innovative evaluation of local injective gel of curcumin on the orthodontic tooth movement in rats. Dent Res J 2018; 15(1): 40-49.

12. Cai Z, Feng Y, Li C, Yang K, Sun T, Xu L, et al. Magnoflorine with hyaluronic acid gel promotes subchondral bone regeneration and attenuates cartilage degeneration in early osteoarthritis. Bone 2018; 116(1): 266-278.

13. Fernandes MT, Fernandes KB, Anibal FF, Shimoya-Bittencourt W, Santos VM, de Oliveira Perrucini PD, et al. Functional status and severity of osteoarthritis in elderly is associated to the polymorphism of TNFA gene. Adv Rheumatol 2019; 59(1): 25-31.

14. Varela-Eirin M, Loureiro J, Fonseca E, Corrochano S, Caeiro JR, Collado $\mathrm{M}$, et al. Cartilage regeneration and ageing: targeting cellular plasticity in osteoarthritis. Ageing Res Rev 2018; 42(2): 56-71.

15. Bert J, Kenney J, Sgaglione NA, Mc Clelland S, Brophy R, Toth $\mathrm{J}$, et al. Viscosupplementation for osteoarthritis of the knee: a key opinion leader panel discussion. J Manag Care Spec Pharm 2018; 24(6-a Suppl): S2-S8.
16. Holyoak DT, Tian YF, van der Meulen MC. Osteoarthritis: Pathology, mouse models, and nanoparticle injectable systems for targeted treatment. Ann Biomed Eng 2016; 44(6): 2062-2075.

17. Hong Z, Gao H, Su Y, Xu B, Wu Z. Effect and mechanism of total alkaloids of strychnine on papain induced rabbit knee osteoarthritis. Biomed Res 2018; 29(8): 1590-1597.

18. Akman YE, Sukur E, Senel A, Oztas Sukur NE, Talu CK, Ozturkmen $Y$. The comparison of the effects of a novel hydrogel compound and traditional hyaluronate following micro-fracture procedure in a rat full-thickness chondral defect model. Acta Orthop Traumatol Turc 2017; 51(4): 331-336.

19. Tamura T, Higuchi Y, Kitamura H, Murao N, Saitoh R, Morikawa $\mathrm{T}$, et al. Novel hyaluronic acid-methotrexate conjugate suppresses joint inflammation in the rat knee: efficacy and safety evaluation in two rat arthritis models. Arthritis Res Ther 2016; 18(1): 79-85.

20. Ozkan FU, Uzer G, Türkmen I, Yildiz Y, Senol S, Ozkan K, et al. Intra-articular hyaluronate, tenoxicam and vitamin $\mathrm{E}$ in a rat model of osteoarthritis: evaluation and comparison of chondroprotective efficacy. Int J Clin Exp Med 2015; 8(1): 1018-1026. 